\title{
Breaking the Barriers to Access a Low Cost Intra-Dermal Rabies Vaccine through Innovative "Pooling Strategy"*
}

\author{
Omesh Kumar Bharti ${ }^{1}$, Wim Van Damme ${ }^{2}$, Kristof Decoster ${ }^{2}$, Petros Isaakidis ${ }^{2}$, An Appelmans ${ }^{2}$, \\ Vidya Ramachandran ${ }^{3}$, Archana Phull ${ }^{4}$ \\ ${ }^{1}$ Government of Himachal Pradesh, Shimla, India; ${ }^{2}$ Institute of Tropical Medicine, Antwerp, Belgium; ${ }^{3}$ National Institute of Epide- \\ miology, Chennai, India; ${ }^{4}$ Bureau Chief Daily Post, Shimla, India. \\ Email: bhartiomesh@yahoo.com
}

Received March $7^{\text {th }}, 2012$; revised April 10 ${ }^{\text {th }}, 2012$; accepted May $12^{\text {th }}, 2012$

\begin{abstract}
Background: In India every year an estimated 20,000 patients die of Rabies. Major reason for poor compliance to anti-rabies prophylaxis is the high cost of anti-rabies vaccine being prescribed intramuscularly (IM) as a routine i.e. 44.5 USD per course of five injections. In 1992 WHO recommended low cost intra-dermal rabies vaccination (IDRV), which costs only 7.5 USD or less per animal bite course. Methods: Interviews with doctors revealed that they were not prescribing intra-dermal anti-rabies vaccination as they were either not aware or were not confident of this route of rabies vaccination. Also the vaccine vial did not have the label for "intra-dermal use". These barriers were removed by advocacy efforts with policy makers \& drug companies, credit sharing \& team building, which led to starting of first intra dermal anti-rabies clinic of North India on $2^{\text {nd }}$ August 2008. Results: Within a month of start of intra-dermal rabies vaccination clinic, i.e. by $2^{\text {nd }}$ September, 2008, there was an increase in the hospital patient load by 2.8 times, and poor patients load by 3.2 times. In just less than two-year time, 200,000 USD of poor patients were saved and 5769 patients vaccinated. Each patient was asked to bring one vial on first visit \& rest of doses were given "free" by pooling strategy. Pooling strategy involved distribution of one vial of vaccine among four persons and keep the three vials for use one by one by all the four patients on subsequent three visits. Another offshoot of the strategy was to prevent wasting of even few drops of vaccine that used to remain in each vial of $1 \mathrm{ml}$ after distribution among four patients $(0.2 \mathrm{~mL}$ or less). Out of more than 5000 vials utilised, every time we would transfer the left out drops of vaccine to the next new vial and use it immediately on a new pool of patients waiting for vaccination. We would, however, discard the unused vaccine after eight hours of reconstitution at the end of the day. The vaccine so saved turned to be a stock of more than 100 vials in less than two years that we were able to give free to more than 225 rag pickers, garbage collectors and newspaper hawkers on World Rabies Day, Sep 28, 2010. Conclusions: With intra-dermal clinic, we were able to successfully introduce the new cost effective intra-dermal method of rabies vaccination despite all odds \& vested interests of companies \& old mindset of doctors that had blocked this technique till now. This will go a long way in reducing the burden of disease \& death due to rabies from India.
\end{abstract}

Keywords: Intra-Dermal Route for Anti-Rabies Vaccination; Rabies

\section{Introduction}

Rabies has been one of the most dreaded diseases since centuries leading to painful death in human beings. It poses a potential threat to 3.3 billion people annually. Globally around 55,000 people die of rabies [1] yearly and out of them 20,000 die in India alone. Deaths due to rabies are responsible for 1.74 million DALYs lost each year. An additional 0.04 million DALYs are lost through mortality and morbidity due to side effects of nerve-tissue vaccine (NTV) [1]. The annual estimated cost of rabies is USD 583.5 million [1]. About USD 400 million

*Competing interests: none. can be saved if we shift from NTV to cell culture vaccines (CCV) all over Asia and Africa, while 1.78 million DALYs can be averted [1].

"In India, the animal bite incidence rate is 17.4 million bites every year. The frequency of animal bite is 1 every 2 seconds and that of death is 1 in 30 minutes. The annual medicinal cost (vaccines + other drugs) for treatment of animal bite is INR 2 billion (4.4 million USD) and annual man days lost due to animal bite is INR 38 million” [2].

The discovery of NTV in 1885 by Dr. Louis Pasteur served the purpose of saving lives for 125 years and it is still being used in many countries [3] though WHO does 
not recommend its use now [4]. The NTVs consisted of $5 \%$ suspension of infected animal nervous tissue which had been inactivated [5]. The modern cell culture antirabies vaccines (CCV) are manufactured through neutralizing different cell lines infected with rabies virus with different modern methods [6].

The cost of one complete course of CCV is around USD 44.5 when given through an intramuscular (IM) route and comes down to USD 7.5 or less if administered through an intra-dermal route (ID) [7]. This cost cutting results from the fivefold reduction of vaccine volume in the ID route. However, we need to share the vial that is made for IM use, as single dose formulations for ID are not yet available.

WHO recommended the use of a cost effective intradermal regimen (IDRV) in 1992 [8] and it was introduced in Thailand, Philippines and subsequently in Sri Lanka by 1996. Many Non Governmental Organizations (NGOs) demanded that the IDRV be started in India as well. But due to different reasons, this could not materialize.

NTV vaccine used to be given free to all in India, but it was stopped by the Supreme Court in 2004 citing some rare adverse reactions, occurring in 1:5000 to $1: 12,000$ vaccine doses [9]. The ban on NTV was sudden. However, with NTV unavailable, and the new CCV vaccine unaffordable, many patients were left in the lurch. We observed in our clinical practice that some patients, who could not afford CCV, died of rabies. One such recent report was received in December 2009 from district Hamirpur [10]. In another incident, a household had to borrow USD 1000 to vaccinate all household contacts after a death due to rabies in the family in the remote Sirmour district of Himachal Pradesh.

The activists, who helped vaccine companies put up a strong case in the Supreme Court of India for a ban on NTV, did not bother either to look for an affordable alternative for the patients or ask these vaccine companies to allow intra-dermal use of vaccine, like in other countries.

This all led to acute shortages of the CCV across India, that could have been avoided had the intra-dermal use become a routine practice before the NTV was banned.

When the pressure to start IDRV mounted, the drug regulatory authorities, Drug Controller General of India (DCGI) ordered that the trials for efficacy and effectiveness of intra-dermal rabies vaccine should be done in India before any approval for IDRV could be given. The Indian government ordered the Indian Council for Medical Research in 2003 to conduct trials that proved its efficacy [11].

The DCGI then granted approval for IDRV subject to the condition that clinics with minimum 50 patients daily can start IDRV and vaccine vial label should mention "for IM/ID use”, which no vaccine manufacturer was ready to do, fearing losses. Even the government vaccine manufacturers refrained from following the order as there was no compulsion to do so. Later this limit of 50 patients was lowered to 10 , and subsequently it was abolished under pressure from some NGOs like Jan Swasthya Abhiyaan etc.

\section{Method}

Meanwhile, we had contacted a vaccine company that mentioned "for IM/ID use" on the label to supply ten vials of vaccine and we announced free IDRV to those who wished to opt for this. Three poor patients opted for IDRV, and the first intra-dermal clinic of the North-West of India was born at Shimla, not in a premier medical college but in a small referral hospital.

The poor people, for whom the intramuscular vaccine was earlier unaffordable at a price of 44.5 USD, could now come to the clinic for low cost intra-dermal rabies vaccination with single vaccine vial to be purchased for pooling, costing only 7.5 USD.

Another issue was to share the vaccine $(1 \mathrm{~mL} / 0.5 \mathrm{~mL}$ vial), as only $0.2 \mathrm{~mL}$ vaccine was required per patient per visit and vial volume was $1 \mathrm{~mL}$ or $0.5 \mathrm{~mL}$. The issue was resolved by pooling the patients and the vials. All nearby hospitals were requested to send patients to a central place for vaccination after wound washing and first aid. A patient was asked to bring one vial of vaccine when he first reports to the clinic and rest of doses are given "free" on subsequent three visits.Pooling strategy involved distribution of one vial of vaccine among four persons and keep the three vials for use one by one by all the four patients on subsequent three visits. This "pooling technique" helped us to avoid vaccine wastage and pass on the cost benefit to the patients. This helped in easy availability of vaccine in the market, as less vaccine was required in the ID clinic for the same number of patients.

\section{Results}

A month after the introduction of the new ID technique at the Shimla ID clinic, the hospital patient load had increased by 2.8 times, and that of poor patients by 3.2 times. The regimen approved by DCGI is updated Thai regimen of administration of $0.1 \mathrm{~mL}$ vaccine on both the deltoids on day $0,3,7$ and 28 .

\section{The Innovation}

Another offshoot of the strategy was to prevent wasting of even few drops of vaccine that used to remain in each vial of $1 \mathrm{~mL}$ after distribution among four patients $(0.2$ $\mathrm{mL}$ or less). Out of more than 5000 vials utilised in less than two years, every day we would transfer the left out drops of vaccine to the next new vial and use it immedi- 
ately on a new pool of patients waiting for vaccination. We would, however, discard the unused vaccine after eight hours of reconstitution at the end of the day. We did take all precautions to maintain the sterility by aseptic no-touch technique and maintained cold temperature (2 - 8 degree Celsius) of the vaccine with the help of a fridge. The vaccine so saved became a stock of more than 100 vials in less than two years that we were able to give free to more than 225 rag pickers, garbage collectors and newspaper hawkers on World Rabies Day, Sep 28, 2010. In 2008, before the start of IDRV in the month of October, the Chief Medical Officer of district Shimla had spent the entire amount of USD 60,000 available for emergency medicine purchase for buying anti-rabies vaccines. But after the IDRV clinic started, he utilized the money, thus saved, for other essential medicines for the poor. Gradually, the technique was replicated in other hospitals in Himachal.

In less than two years, we have vaccinated 5769 animal bite victims in Himachal using insulin syringes and needles without any failure, even in cases of proven rabid dog bite victims. Around 12,000 vials of ARV vaccine were given, saving more than USD 200,000.

\section{Expanding the Innovation}

Already nine states have started IDRV in India. If IDRV is implemented in the entire country, it will save much money and many lives. A positive sign is that with many states opting for the ID route, pressure has mounted on the vaccine companies to come forward to write "for IM/ID use" on the label [12].

As part of an awareness campaign for the public, we also developed a poster after performing a knowledge gap analysis in English and in colloquial Hindi.

Now we are advocating with policy makers in neighboring countries that are still using NTV or are not using the ID route, to adopt this low cost method. Our efforts have yielded some results in Indonesia, where a number of rabies deaths were reported in 2010.

We demonstrated in the small hill state of Himachal that easy availability, affordability and information education and communication (IEC) campaigns about the importance of "wound wash and antiseptics" can reduce deaths to almost zero over a three year period. There were nine deaths reported due to rabies in Himachal in 2008, 4 in 2009 and no death was reported in 2010.

\section{The Way Forward}

We need to explore low cost methods like 4 site 3 visit ID schedules [13] of IDRV and also to establish clearly if there is any danger to those who consume raw milk of rabid cow [14], as this has often forced people to get anti-rabies prophylaxis by borrowing. Combining intra dermal ARV with Hepatitis-B or DPT in Africa and Asia are other possibilities to explore; they need more research though.

\section{More Vaccines Can be Low Cost}

Research has demonstrated that there is a potential for low cost vaccination through the intra dermal route for ten diseases including injectable polio vaccine, IPV [15] and this can directly benefit the poor patients all over the world and spare money for procuring other drugs for the poor. But barriers, like the ones enumerated above, exist and determined action is needed to overcome them. Intra dermal use is not only useful for reducing costs but also for dose sparing in case of scarcity of vaccines as was the case with the recent H1N1 pandemic [16].

\section{Conclusion}

With the intra-dermal clinic, we were able to successfully demonstrate how to implement a low cost technology despite vested interests of companies \& the stubborn mindset of doctors that had blocked this technique for long, despite recommendations of WHO.

\section{Acknowledgements}

We acknowledge Ms. Dropda Shyam, a Female Health Worker then working at Shimla Clinic, for her suggestions in developing and fine-tuning the pooling technique; and Dr. S. L. Chaudhary, Pediatrician, for assuring patients regarding the efficacy of the new technique of intra dermal vaccination while we first started the clinic.

\section{Funding Support}

The funders, (DGD) had no role in study design, data collection and analysis, decision to publish, or preparation of the manuscript.

"We acknowledge support from the 'Emerging Voices for Global Health' venture at the Institute of Tropical Medicine (ITM), Antwerp, Belgium. The Emerging voices endeavour started as an essay competition in April 2010 and was financed by the Framework Agreement between ITM and the Belgian Development Cooperation (DGD). More detail on the Emerging voices project, winners and coaching process can be found on the following website: http://www.itm.eu/colloq2010".

\section{REFERENCES}

[1] D. L. Knobel, S. Cleveland, P. G. Coleman, E. M. Fèvre, M. I. Meltzer, et al., "Re-Evaluating the Burden of Rabies in Africa and Asia," Bulletin of the World Health Organization, Vol. 83, No. 5, 2005, pp. 360-368.

[2] M. K. Sudershan, “Assessing Burden of Rabies in India 
WHO Sponsored National Multi-Centric Survey 2003,” Association for Prevention and Control of Rabies in India, AMS Code 1079420, 2004.

[3] T. Burki, “The Global Fight against Rabies,” The Lancet, Vol. 372, No. 9644, 2008, pp. 1135-1136. doi:10.1016/S0140-6736(08)61462-2

[4] World Health Organization, "WHO Expert Consultation on Rabies: First Report," World Health Organization, Geneva, 2005 (WHO Technical Report Series, No. 931).

[5] S. D. Semple, "Scientific Memoirs by Officers of the Medical and Sanitary Departments of the Government of India,” No. 44, New Series, Calcutta, 1911.

[6] S. Madhusudana, "Rabies," Mc Millan Medical Communications, Gurgaon, 2011, p. 151.

[7] Average Prices in India 2010 as Reference.

[8] World Health Organization, "WHO Expert Committee on Rabies, 8th Report,” World Health Organization, Geneva, 1992.

[9] S. Madhusudana, "Rabies,” Mc Millan Medical Communications, Gurgaon, 2011, p. 150.

[10] Departmental Communication from Chief Medical Officer, Unpublished Document, Hamirpur, 2009.

[11] "Multicentric Study on the Use of Intradermal Administration of Tissue Culture Anti-Rabies Vaccines in India,”
Web Document, pp. 12-15.

http://icmr.nic.in/annual/2004-05/nie/clinical_trials.pdf

[12] Rabipur, Virorab and Indirab Brands in India Allow Intra-Dermal Use Apart from Intramuscular Use and Write "For IM/ID Use".

[13] M. J. Warrell, A. Riddell, L.-M. Yu, J. Phipps, L. Diggle, et al., "A Simplified 4-Site Economical Intradermal PostExposure Rabies Vaccine Regimen: A Randomised Controlled Comparison with Standard Methods," PLOS Neglected Tropical Diseases, Vol. 2, No. 4, 2008, p. e224. doi:210.1371/journal.pntd.0000224

[14] Centers for Disease Control and Prevention, "Mass Treatment of Humans Who Drank Unpasteurized Milk from Rabid Cows-Massachusetts, 1996- 1998,” MMWR Weekly, Vol. 48, No. 11, 1999, pp. 228-229.

[15] PATH, "Intradermal Delivery of Vaccines: A Review of the Literature and Potential for Development for Use in Low- and Middle-Income Countries," Program for Appropriate Technology in Health (PATH), Seattle, 2009.

[16] J. K. Hickling, K. R. Jones, M. Friede, D. Zehrung, D. Chen and D. Kristensen, "Intradermal Delivery of Vaccines: Potential Benefits and Current Challenges," Bulletin of the World Health Organization, Vol. 89, 2011, pp. 221-226. doi:10.2471/BLT.10.079426 